\title{
Comparisons in Outcome and Subject Comfort between Rotation Chair Systems
}

\author{
Bong Jik Kim ${ }^{1,2}$, Yu-Kyung Won ${ }^{1}$, Jaihwan Hyun ${ }^{1}$, Woo-Sung Na ${ }^{1}$, \\ Jae Yun Jung ${ }^{1}$, and Myung-Whan Suh ${ }^{3}$ \\ ${ }^{1}$ Department of Otolaryngology Head \& Neck Surgery, Dankook University Hospital, Cheonan, \\ ${ }^{2}$ Wide River Institute of Immunology, Seoul National University College of Medicine, Seoul, \\ ${ }^{3}$ Department of Otorhinolaryngology-Head and Neck Surgery, Seoul National University Hospital, Seoul, Korea
}

Received March 1, 2017

Revised April 11,2017

Accepted May 15, 2017

\section{Address for correspondence Myung-Whan Suh, MD, PhD \\ Department of Otorhinolaryngology- Head and Neck Surgery, \\ Seoul National University Hospital, \\ 101 Daehak-ro, Jongno-gu, \\ Seoul 03080, Korea \\ Tel $+82-2-2072-4845$ \\ Fax +82-2-766-2447 \\ E-mail drmung@naver.com}

\begin{abstract}
Background and Objectives: A rotation chair test has been used to evaluate the function of the horizontal semicircular canals. Currently, two chair systems according to the presence of cylindrical darkroom are used in a clinic setting. However, it has not been thoroughly investigated whether one system is superior to the other system or not. In this study, we aimed to compare test outcomes and subject convenience between two systems. Subjects and Methods: Twenty subjects with no history of otologic disease were enrolled. Subjects were tested with two systems: system $[\mathrm{A}]$ with a cylindrical chamber and system $[\mathrm{B}]$ with no chamber. The results of sinusoidal harmonic acceleration (SHA), step velocity (SV), and visua fixation (VFX) tests were compared between the systems. Subject convenience was assessed with a questionnaire survey and results were compared between the systems. Results: There were no significant differences in gain or asymmetry in SHA test between the systems. However, the phase of system [A] was significantly lower than that of system $[B]$ at $0.16 \mathrm{~Hz}$. There was no significant difference between the systems in directional preponderance (DP) gain or DP time constant. Regarding the VFX test, gain was higher in system [A] than system $[B]$. Subjects reported less stuffiness and less anxiety with system $[B]$ than system $[A]$, while preferring the system $[A]$ goggles. Conclusions: A rotation chair system without a darkroom can provide a more comfortable experience for subjects in terms of stuffiness and anxiety, while showing comparable results in SHA and SV tests with a darkroom system.

J Audiol Otol 2017;21(2):88-94
\end{abstract}

KEY WORDS: Rotating chair test $\cdot$ Sinusoidal harmonic acceleration $\cdot$ Step velocity . Visual fixation

\section{Introduction}

A rotation chair test is used to evaluate the function of the horizontal semicircular canals through the vestibulo-ocular reflex (VOR) $[1,2]$. To augment the amplitude of the nystagmus and accurately quantify the VOR, inhibition of visual fixation (VFX) during the test is essential [3,4]. There are two different methods to inhibit VFX: 1) putting the subject in a darkroom and 2) covering the eyes of the subject with vari-

This is an Open Access article distributed under the terms of the Creative Commons Attribution Non-Commercial License (http://creativecommons.org/licenses/by-nc/4.0/) which permits unrestricted non-commercial use, distribution, and reproduction in any medium, provided the original work is properly cited. ous devices. A cylindrical darkroom not only blocks vision effectively but is also useful when performing visual-vestibular interaction tests such as visual enhanced VOR or VFX tests. Optokinetic bars can be displayed on the cylindrical wall by means of a lampshade with vertical slits (visual enhanced VOR test). A laser beam mounted on the chair, pointing to the cylindrical wall, can provide a fixation target with constant distance even during chair rotation (VFX test). Lightblocking goggles may be an alternative to a cylindrical darkroom. Considering the large structure of a cylindrical darkroom, goggles would seem to be a practical solution for preventing VFX. However, in terms of visual-vestibular interaction tests, there can be some limitations without a cylindri- 
cal darkroom. Optokinetic bars cannot be displayed in a controlled visual environment and a fixation target at a sufficient distance cannot be presented to the subject. A dark room is quite a burden in terms of space occupation but there seems to be a good reason for the large structure.

Other than space occupation, there may be some other problems with a darkroom. A cylindrical darkroom may increase the price of the system due to the large structure. It is unfortunate that there are patients who cannot undergo a rotation chair test because of the high cost. If the price of the system was cheaper, we may be able to reduce the price of the test and offer the test to more patients who need evaluation. Another shortcoming of a darkroom is that some patients, especially children, are afraid of being alone in an isolated darkroom [5]. Some patients may also refuse the test due to claustrophobia [6]. Ideally, the patient and the tester would be in the same space without walls in between. Even though the patients' eyes are covered with goggles and cannot see anything, patients may feel more comfortable with the thought that they are not isolated nor locked inside a small structure. Patients may feel confident that the tester (or their guardian) can immediately see them and hear them if they call for help. Free verbal communication between the patient and tester without a speakerphone can also be a big relief. Considering these points, it seems that there is tradeoff for a darkroom between more detailed vestibular evaluation (visual-vestibular interaction) and patient comfort.

Currently, there are rotation chair systems without a cylindrical darkroom on the market. These systems tend to be cheaper than conventional systems with a standard darkroom. Also, it can be presumed that these systems without a darkroom may provide more comfortable test conditions, especially for those patients who experience claustrophobia. However, it has not been determined whether such rotation chair systems without a darkroom are truly beneficial in terms of patient compliance in real clinical settings. Also, because patients are blindfolded in both systems, patients may still feel uncomfortable regardless of the darkroom. More importantly, it has not been demonstrated that the test outcomes are the same between the two systems for visual-vestibular interaction tests such as VFX tests. For rotation chair systems without a darkroom, a small LED light inside the goggles can work as a fixation target, instead of a laser beam pointing at the $1 \mathrm{~m}$ distant darkroom walls. The fixation distance would be very short under these conditions. The difference in fixation distance may result in a difference in outcome. In this study, we aimed to compare test outcomes and subject convenience between rotation chair systems with and without a cylindrical darkroom to determine whether one system is su- perior to the other system or not.

\section{Subjects and Methods}

\section{Subjects}

Between 2011 and 2012, 20 healthy normal subjects with no history of otologic disease underwent rotation chair tests with two different systems: system [A] with a darkroom (System 2000; Micromedical Technologies, Chatham, IL, USA) and system [B] without a darkroom (Nydiag 200; Interacoustics, Assens, Denmark) (Fig.1). Of the subjects, 10 (50\%) were men, and $10(50 \%)$ were women. The mean age of the subjects was $32.3 \pm 7.4$ years old.

This study was approved by the Institutional Review Board of Dankook University Hospital (IRB No. 2011-09-0330). Written informed consent was obtained from all participants.

\section{Experimental procedure}

All 20 subjects were tested with both rotation chair systems. The interval between the two tests was from $1 \mathrm{~h}$ to 1 day, and the order of the test systems was randomized. Testing parameters and preparation were the same between the two systems for the sinusoidal harmonic acceleration (SHA) and step velocity (SV) tests. The subject's head was aligned with the chin pitched $30^{\circ}$ nose down, such that the horizontal semicircular canals were in the plane of rotation, and tests were performed with goggles fitted tightly. For all procedures, a calibration was done first. To keep the subjects awake during the tests, subjects were required to count numbers or talk with the tester. For SHA, both eyes were completely covered with the infrared video recording goggles. The chair was rotated at $0.01,0.04$, and $0.16 \mathrm{~Hz}$, with a maximum velocity of $60 \%$ s. Gain, asymmetry and phase were evaluated as outcome measures $[7,8]$. For SV, clockwise and counterclockwise $100 \% \mathrm{~s}^{2}$ acceleration (perrotation) to a target velocity of $100 \%$ and $100 \% \mathrm{~s}^{2}$ deceleration (post-rotation) was performed. Directional preponderance (DP) of the gain and time constant were evaluated as the outcome measures. DP of gain and time constant were defined as $100 \times[$ (Rt. per-rotatory nystagmus + Lt. postrotatory nystagmus)-(Lt. per-rotatory nystagmus + Rt. postrotatory nystagmus)]/(Rt. per-rotatory nystagmus + Lt. postrotatory nystagmus + Lt. per-rotatory nystagmus + Rt. postrotatory nystagmus) [9]. For VFX, the two systems had a slightly different stratagem. System [A] used a laser beam as the fixation target, which was projected from the top of the chair to the wall of the cylindrical dark chamber and the fixation distance was $\sim 1 \mathrm{~m}$. System [B] used LED lights as the fixation target, placed inside each eyepiece of the goggles. The fixation distance was $\sim 2 \mathrm{~cm}$. Other than this, the rotation 
of the chair was identical: the chair was rotated at $0.04 \mathrm{~Hz}$ with a maximum velocity of $60 \%$. The gain was compared between the two systems.

\section{Questionnaires}

To assess subject discomfort, a questionnaire with six questions was completed by the subjects just after each rotation chair test. Because all subjects underwent two tests, all subjects filled out two questionnaires. The questions were, 1) "In general, was this test uncomfortable?", 2) "Did you feel stuffy during the test?", 3) "Were you anxious because you could not see anything?", 4) "Were the goggles uncomfortable?", 5) "Did you feel dizzy during the test?", and 6) "How many dots did you see during the VFX test?". For questions, 1) to 5), the subjects were forced to choose among five answers: 1) Yes, definitely, 2) I think so, 3) So-so, 4) I don't think so, or 5) No, not at all. Question, 6) was a short-answer question, without any examples. A numeric response value was assigned to each response option from 1) to 5) as 1, 2, 3, 4, 5 .

\section{Statistical analysis}

For statistical analysis, the paired t-test was used with the SPSS software (version 19.0; IBM Co., Armonk, NY, USA) to compare the mean values of SHA test results (gain, asymmetry, and phase), mean DP values of SV test results (gain, time constant), VFX gain results, and questionnaires results between the two systems. Differences were considered to be statistically significant if $p<0.05$.

\section{Results}

\section{Sinusoidal harmonic acceleration (SHA)}

There was no significant difference in gain or asymmetry between the systems (Fig. 2). Regarding the phase, there was no significant difference between the two systems at $0.01 \mathrm{~Hz}$ or $0.04 \mathrm{~Hz}$. However, the phase was significantly $(p=0.001)$ greater at $0.16 \mathrm{~Hz}$ with system $[\mathrm{B}]\left(8.0 \pm 5.6^{\circ}\right)$ than system [A] $\left(2.6 \pm 2.7^{\circ}\right)$.

\section{Step velocity (SV)}

The DP of gain was $1.9 \pm 12.9 \%$ with system $[\mathrm{A}]$ and $-7.3 \pm$ $15.5 \%$ with system [B] (Fig. 3). The DP of the time constant was $1.9 \pm 10.4 \%$ with system [A] and $1.2 \pm 11.8 \%$ with system $[\mathrm{B}]$. The DP values were all quite close to 0 (symmetric $\mathrm{SV}$ response) and there was no significant difference between the two systems in terms of DP gain or DP time constant.

\section{Visual fixation (VFX)}

VFX gain was $0.077 \pm 0.019$ (range, $0.04-0.12$ ) with system [A] and $0.000 \pm 0.000$ (range, $0.00-0.00$ ) with system [B]. That is, with system [B], the VFX gain was 0.00 in every subject, without exception. The VFX gain was significantly higher with system [A] than system [B] $(p<0.001)$.

\section{Questionnaires}

Because the questions and examples were all asked in a negative statement, lower values represent unfavorable responses and higher values represent favorable responses. For the question: 1) "In general, was this test uncomfortable?",
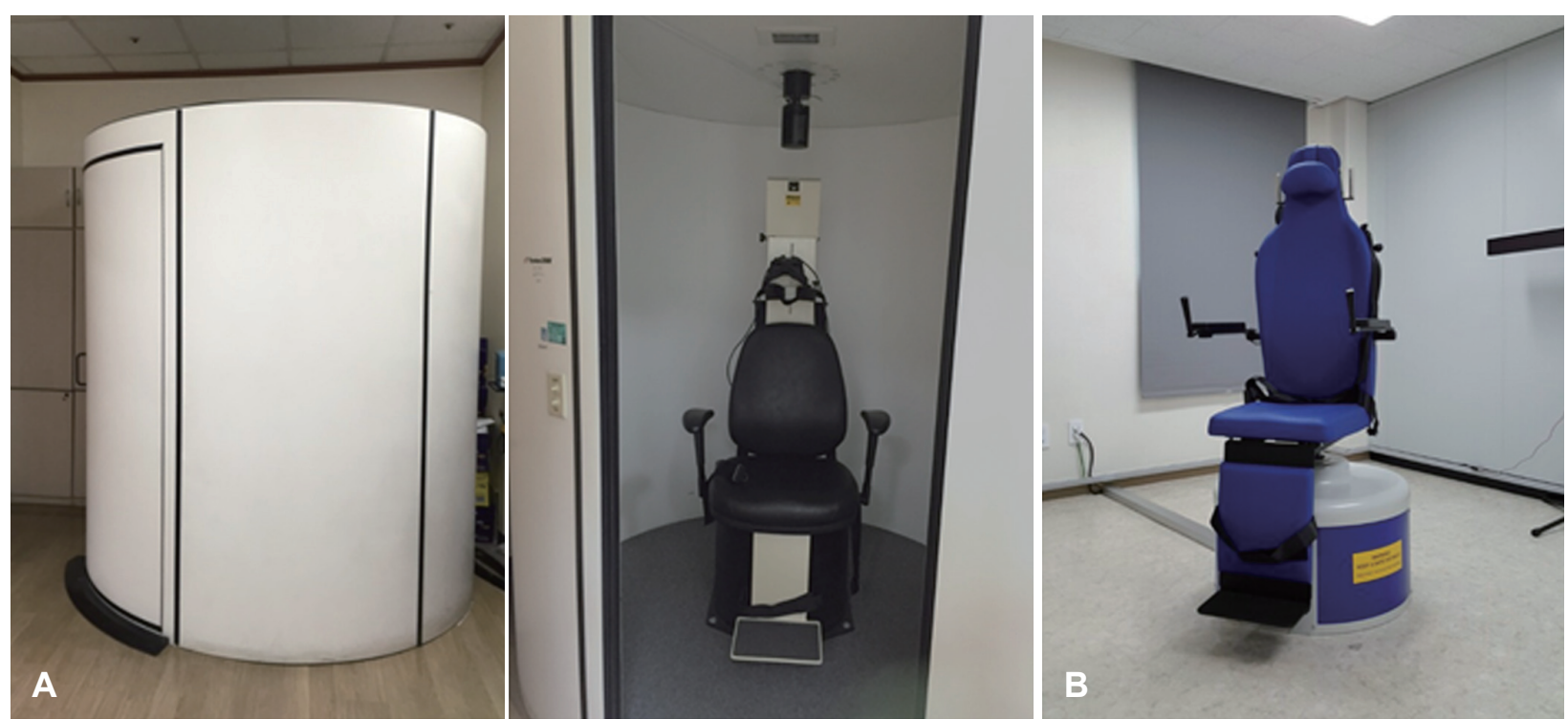

Fig. 1. Two different rotation chair systems. System [A]: System 2000 from Micromedical Technologies with a cylindrical chamber (A), System [B]: Rotary chair Nydiag 200 from Interacoustics without a darkroom (B). 
responses for system [A] (3.7 \pm 0.8$)$ and system [B] $(3.2 \pm 1.1)$ were similar; 2) "Did you feel stuffy during the test?", subjects preferred system $[\mathrm{B}](3.7 \pm 1.1)$ to system $[\mathrm{A}](3.1 \pm 1.1)$ with a significant difference $(p=0.05) ; 3)$ "Were you anxious because you could not see anything?", subjects preferred system [B] $(4.2 \pm 0.7)$ to system [A] $(3.3 \pm 1.4)$ with a significant difference ( $p=0.002) ; 4)$ "Were the goggles uncomfortable?", subjects preferred system [A] goggles $(3.5 \pm 0.8)$ to system [B] goggles (2.5 \pm 1.1$)$, with a significant difference ( $p=0.003)$; $5)$ "Did you feel dizzy during the test?", responses for system [A] $(3.5 \pm 1.1)$ and system [B] $(3.7 \pm 1.2)$ were similar; and 6) "How many dots did you see during the VFX test?", all subjects identified one target with system [A] and all subjects identified two targets with system [B], without exception.
Fig. 2. Results of the SHA test. There were no significant differences in gain or asymmetry between the two systems at any frequencies (A-D). Regarding phase, a significant difference between the two systems was observed at $0.16 \mathrm{~Hz}(p=$ $0.001)(E, F) .{ }^{*} p<0.05$. SHA: sinusoidal harmonic acceleration.
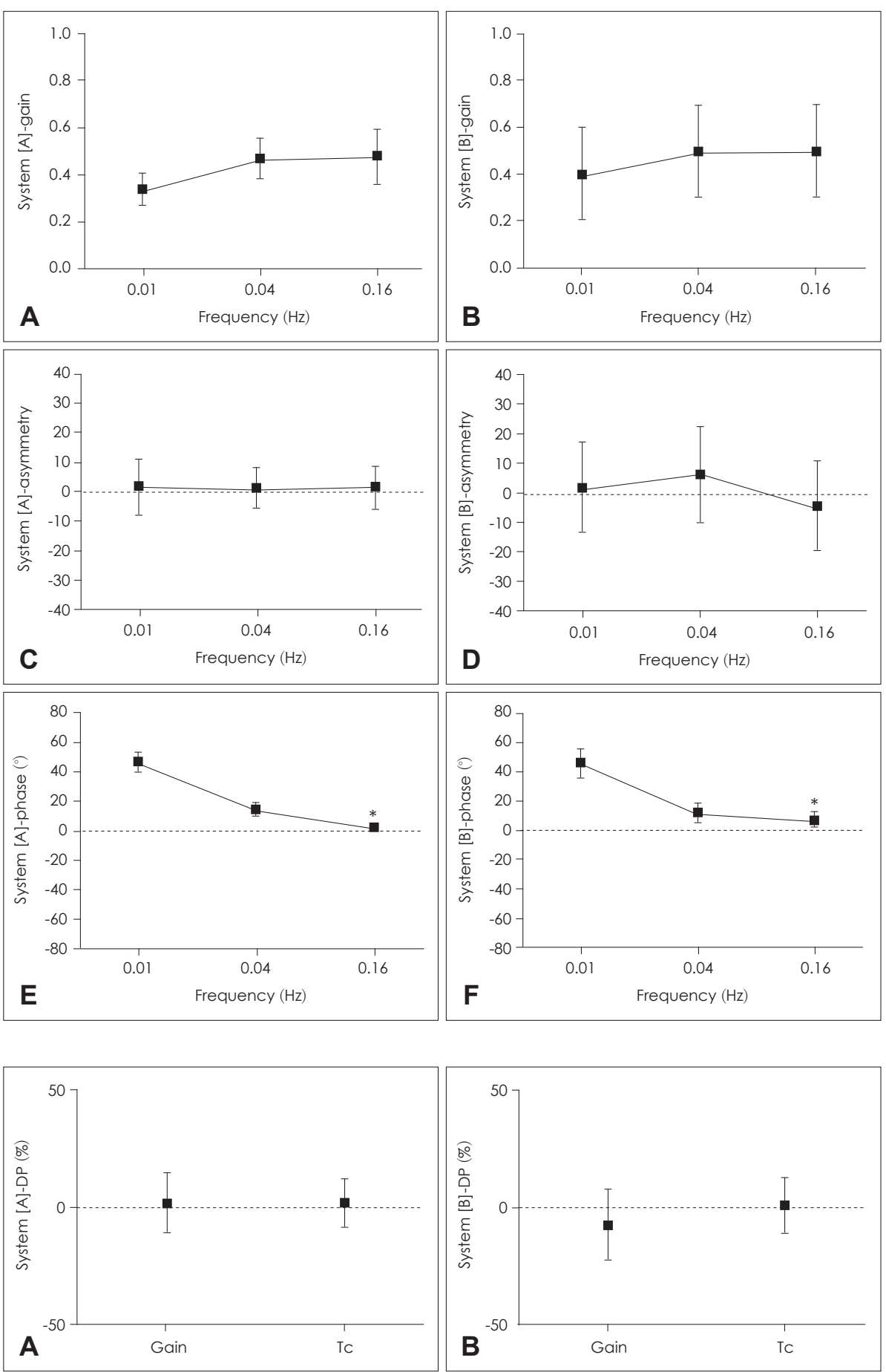

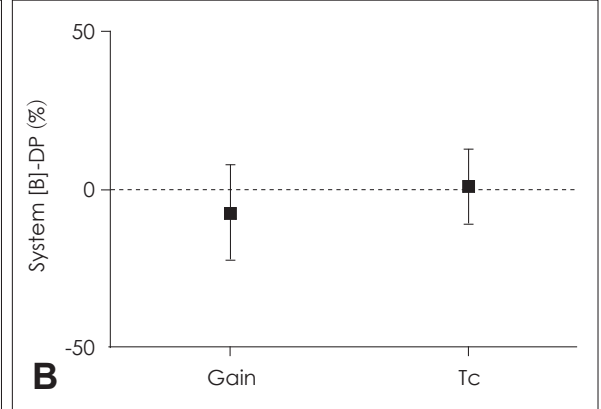

Fig. 3. Results of the SV test. There were no significant differences in the DP of gain or time constant (TC) between the two systems (A, B). SV: step velocity, DP: directional preponderance. 


\section{Discussion}

When we compared the two most frequently used tests (SHA and SV) between the two rotation chair systems, there were minimal differences. For the SHA test, gain, phase, and asymmetry were analyzed and there were no significant differences between the two systems, except for phase at 0.16 $\mathrm{Hz}$. The $0.16 \mathrm{~Hz}$ phase was higher with system [B], but the difference was small $\left(<5.5^{\circ}\right)$ and both values were within the normal limits provided by the manufacturers. Although the difference was statistically significant, it seems that it was likely not clinically relevant. For the SV test, the mean DPs of gain and time constant approached 0 with both systems. On the individual level, the DPs of gain and time constant were also similar between the two systems in each subject. Considering that SHA and SV tests are performed in complete darkness, it seems reasonable that we found no difference in the outcome regardless of the presence or absence of the darkroom. That is, it does not matter which covering method is used (darkroom or goggles), if we can completely block visible light during SHA and SV tests. We may now compare the two systems in terms of other visual-vestibular interactions and patient compliance, because the performance and main outcomes were essentially the same.

In the VFX test, the gain of system [A] was significantly higher than that of system [B]. That is, when recorded with system [A], a small but identifiable nystagmus was detected even while the subject was fixating his or her visual focus on the target. In contrast, the nystagmus was completely abolished (gain 0.0 in every subject) when the subject was fixating his or her visual focus on the target with system [B]. It is not clear why the VFX gain was so low with system [B], but we assume that the distance from the eye to the target is one important reason. It is known that a higher VOR gain is needed to visually fix on a near distance earth-fixed target $[10,11]$. Conversely, if the target is head-fixed, a higher suppression of VOR is needed to prevent the eyes from drifting from the near target: gain of VFX must be lower to fixate on a near target. It seems that the VOR suppression was stronger with system [B], because the distance from the eye to the visual target was much closer ( $\sim 2 \mathrm{~cm}$ distance) compared with system [A] ( 1 m distance). A second hypothesis is that each eye fixed to two different targets may induce a disconjugated eye movement and a difference in VFX gain. As has been mentioned in the results, all the subjects noticed two lights when the visual target was inside the goggles. Third, system [B] may have been unable to detect and record very small nystagmi. Because the two systems have differently shaped goggles, infrared cameras, and operating software, the per- formance in detecting small nystagmi may differ. However, we believe this is not likely. When the raw data of eye movements were inspected manually, we were not able to identify robust nystagmus with system [B]. Also, the SHA and SV test results were nearly identical between the two systems. Because the VFX test is based on the SHA test, the difference in performance between the two systems may not fully explain the difference in VFX outcome.

Very low gain in the VFX test may result in low sensitivity in detecting patients with central problems. In normal subjects without central problems, low gain $(<0.2)$ in the VFX test is a typical finding. Accordingly, very low gain in a VFX test may not be a problem in normal subjects: the specificity of the VFX test will be acceptable. However, it is unclear if the sensitivity of the VFX test will be acceptable when the fixation target is inside the goggles and the VFX gain is so low. Due to the strong suppression of VOR, it is likely that the gain will be very low (normal) even in patients who have a problem in the cerebellum and tend to fail in fixation suppression $[3,12]$. This may result in poor sensitivity in detecting patients with central problems. However, as all the subjects in this study were normal controls, we currently have no data that directly supports this. To confirm our hypothesis, we need to perform a similar study with patients who have a problem in the cerebellum and tend to fail in fixation suppression. It seems that the clinical implications of the difference in VFX gain between the two systems need further study.

According to the questionnaire survey, subjects felt less stuffy and less anxious with the rotation chair system that did not have a darkroom. Despite the fact that the subjects are blindfolded anyway, it seems that the darkroom does cause additional discomfort to the subjects. It is intuitive to understand why the subjects feel more discomfort when the darkroom is present. Entering into a small chamber, closing the door, and being acoustically isolated may cause additional fear. In this study, all subjects were healthy adults and no one gave up the test due to claustrophobia. However, if the subjects were children or claustrophobic, we do think a system without the darkroom will be clinically beneficial. Someone may argue that the difference in discomfort may be due to the subjective wearing sensation of the goggles rather than the darkroom. To verify this, we also asked if the subjective wearing sensation of the goggles was comfortable for the two systems. If the subjective wearing sensation of the goggles was better with system [B], we might have been confused as to whether the stuffiness/anxiety was due to the difference in the goggles or due to the darkroom. However, we found that the subjective wearing sensation of the goggles was better with system [A]. This may indicate that the stuffiness and anxiety 
were more likely due to the darkroom itself.

This study has several limitations. First, it was performed only on subjects with normal vestibular function. Because we derived the results from normal subjects, it may be misleading to extrapolate to patients with vestibular function deficits. Additionally to evaluate the clinical implications of the very low gain in the VFX test, we must test patients with cerebellar disorders. We hope to perform a study with such patients in the near future. Second, if a pure effect of the darkroom is to be evaluated, we should have used the same rotation chair system and only changed the presence/absence of the darkroom. In this study, the rotation chair systems differed as well as the presence/absence of the darkroom. Accordingly, it may be slightly ambiguous if the difference in outcome is due to the darkroom or the difference in the rotation chair system itself. We did think of using the same chair system and simply removing the darkroom for a fair comparison. However, this did not seem clinically relevant, because the manufacturer did not design the system to be used in this manner and no clinician would actually remove the darkroom for this purpose. Also, the fixation targets within the goggles are only optimized for the rotation chair system without a darkroom. Clinically, it would be more informative to compare a system that has been designed to have a darkroom and a system that has been designed not to have a darkroom. We presumed that the effect of a darkroom can be compared between two different systems if the SHA and SV results were generally identical. As this was the case in this study, we believe the differences in VFX gain and the questionnaire survey are mostly due to presence/absence of the darkroom. Lastly, we have only tested $0.01,0.04,0.16 \mathrm{~Hz}$ due to practical issues. In our routine practice, we usually tested only three frequencies to save time. In order to precisely reflect the routine practice in this study, we have tested these three frequencies as previously used $[13,14]$. Our rationale for this practice is that the sensitivity of SHA is higher in low frequency stimulation, especially for the phase [15-17]. Therefore, we thought it would not undermine the main idea of this study to test three frequencies of SHA, with each representing low frequency (0.01), mid low frequency that is apart from 0.01 (0.04), and mid high frequency that is apart from 0.04 (0.16). However, it would have been best if we have included higher frequencies including 0.32 and $0.64 \mathrm{~Hz}$.

From this study, we were able to demonstrate that a rotation chair system without a darkroom can provide a more comfortable experience for subjects in terms of stuffiness and anxiety. We think this advantage will be clinically beneficial to timid children and adult patients who are claustrophobic. If the price of the rotation chair system and the test charges can be reduced by omitting darkroom installation, patients who cannot afford the expensive test will also benefit. For the clinic, a rotation chair system without a darkroom may take up a smaller space than a system with a large darkroom. However, it should also be mentioned that test outcomes may differ between the two systems. Some visual-vestibular interaction tests such as the visual enhanced VOR cannot be performed when a darkroom is not present. Also, for the VFX test, the gain was significantly lower when there was no darkroom and the fixation target was inside the goggles. We think this low gain may be a problem in detecting patients with central disorders. Taken together, we propose that a rotation chair system without a darkroom can be alternatively used to evaluate the vestibular function, while providing a comfortable experience for subjects and showing comparable results in SHA and SV tests compared with a darkroom system.

\section{Acknowledgments}

This study was supported by the Korean National Research Foundation (NRF-2015R1A2A2A04003938).

\section{Conflicts of interest}

The authors have no financial conflicts of interest.

\section{REFERENCES}

1) Baloh RW, Sills AW, Honrubia V. Impulsive and sinusoidal rotatory testing: a comparison with results of caloric testing. Laryngoscope 1979;89:646-54.

2) Baloh RW, Jacobson KM, Beykirch K, Honrubia V. Horizontal vestibulo-ocular reflex after acute peripheral lesions. Acta Otolaryngol Suppl 1989;468:323-7.

3) An SY, Kim BJ, Suh MW, Rhee CK, Jung JY. Clinical roles of fixation suppression failure in dizzy patients in the ENT clinic. Acta Otolaryngol 2014;134:1134-9.

4) Jung S, Jung JY, Rhee CK, Suh MW. Competence in suppressing the visual fixation among different types of frenzel glasses. Res Vestib Sci 2010;9:134-8.

5) Chou IJ, Tench CR, Gowland P, Jaspan T, Dineen RA, Evangelou N, et al. Subjective discomfort in children receiving $3 \mathrm{~T}$ MRI and experienced adults' perspective on children's tolerability of 7 T: a crosssectional questionnaire survey. BMJ Open 2014;4:e006094.

6) Cyr DG, Moller CG, Moore GF. Clinical experience with the lowfrequency rotary chair test. Semin Hear 1989;10:172-90.

7) Clarke AH. Perspectives for the comprehensive examination of semicircular canal and otolith function. Biol Sci Space 2001;15:393400.

8) O'Leary DP, Davis LL. High-frequency autorotational testing of the vestibulo-ocular reflex. Neurol Clin 1990;8:297-312.

9) Jongkees LB, Philipszoon AJ. Electronystagmography. Acta Otolaryngol Suppl 1964;189:SUPPL 189:1+.

10) Paige GD, Telford L, Seidman SH, Barnes GR. Human vestibuloocular reflex and its interactions with vision and fixation distance during linear and angular head movement. J Neurophysiol 1998;80:2391404.

11) Baloh RW, Beykirch K, Honrubia V, Yee RD. Eye movements induced by linear acceleration on a parallel swing. J Neurophysiol 1988; 60:2000-13.

12) Kim HA, Yi HA, Lee H. Failure of fixation suppression of spontane- 
ous nystagmus in cerebellar infarction: frequency, pattern, and a possible structure. Cerebellum 2016;15:182-9.

13) Jung JY, Kim YH, Suh MW. Difference in the nature of dizziness between vestibular neuritis and sudden sensorineural hearing loss with vertigo. Otol Neurotol 2012;33:623-8.

14) Lee HJ, Jung SD, Jung JY, Suh MW. Significance of correcting the spontaneous nystagmus in siusoidal harmonic acceleration test. Korean J Otorhinolaryngol-Head Neck Surg 2011;54:761-5.
15) Myers SF. Patterns of low-frequency rotational responses in bilateral caloric weakness patients. J Vestib Res 1992;2:123-31.

16) Baloh RW, Honrubia V, Yee RD, Hess K. Changes in the human vestibulo-ocular reflex after loss of peripheral sensitivity. Ann Neurol 1984;16:222-8.

17) Jenkins HA, Honrubia V, Baloh RH. Evaluation of multiple-frequency rotatory testing in patients with peripheral labyrinthine weakness. Am J Otolaryngol 1982;3:182-8. 